\title{
A STUDY OF THE ANTIOXIDANT EFFECT OF ALPHA LIPOIC ACIDS ON SPERM QUALITY
}

\author{
Siti Fatimah Ibrahim, ${ }^{\text {I Khairul Osman, }}$, Srijit Das ${ }^{\text {III, }}$ Abas Mazni Othman, ${ }^{\text {IV }}$ \\ Norzaiti Abdul Majid, ${ }^{\mathrm{V}}$ Mohd Padzil Abdul Rahman ${ }^{\mathrm{IV}}$
}

doi: $10.1590 / \mathrm{S} 1807-59322008000400022$

Ibrahim SF, Osman K, Das S, Othman AM, Majid NA, Rahman MPA. A study of the antioxidant effect of alpha lipoic acids on sperm quality. Clinics. 2008;63:545-50.

OBJECTIVE: Assisted reproductive techniques are useful in helping infertile couples achieve successful conception. Initial studies have shown that sperm cryopreservation, one step in assisted reproduction, causes a dramatic reduction in sperm quality. This has been attributed to, among other things, free radical activities. The aim of the present study was to minimize this oxidative attack by adding an antioxidant into the sperm microenvironment. Alpha lipoic acids were selected for this purpose for their efficient free radical scavenging properties and solubility in lipid and aqueous phases.

METHODS: For this investigation, semen from six Boer bucks was pooled. Seminal analysis of the baseline prior to incubation of samples with different concentrations of Alpha lipoic acids $(0.00625,0.0125,0.025,0.05,0.1 \mathrm{mmol} / \mathrm{ml})$ was performed, and postseminal analysis was conducted after a one-hour incubation. The comet assay was used to observe the effect of Alpha lipoic acids on sperm DNA integrity. Statistical analysis using an unpaired t-test with a significance level of $\mathrm{p}<0.05$ was then performed.

RESULTS: Our results indicate that the sperm motility rate was improved after incubation with Alpha lipoic acids at a concentration of $0.02 \mathrm{mmol} / \mathrm{ml}$. This concentration was also capable of reducing DNA damage.

CONCLUSION: In conclusion, Alpha lipoic acids renders cryoprotection to sperm, thereby improving sperm quality.

KEYWORDS: Sperm. Semen. Action. Antioxidant. Oxidative stress. Alpha lipoic acids. Analysis.

\section{INTRODUCTION}

Infertility is defined as conception failure following regular sexual activities in the absence of anti-contraceptive means for at least one year. ${ }^{1}$ This is a global problem affecting $15-20 \%$ of couples throughout their reproductive life, and its prevalence has been found to increase with age. Causes of infertility in couples are numerous and varied, but the majority are attributable to the male partner. Currently,

\footnotetext{
${ }^{\text {I }}$ Universiti Kebangsaan Malaysia - Physiology, Fakulti Perubatan Malaysia .

II Universiti Kebangsaan Malaysia - Forensic Science Unit, FSKB Malaysia.

III Universiti Kebangsaan Malaysia - Anatomy, Faculty of Medicine Malaysia.

IV MARDI - Molecular Biology and Genetic Engineering Biotech Centre - Malaysia.

${ }^{v}$ Universiti Kebangsaan Malaysia - Biomedical Sciences - Malaysia. Email: timi@medic.ukm.my

Received for publication on April 04, 2008

Accepted for publication on May 23, 2008
}

the important causes of male infertility include azoospermia, oligospermia or hypogonadism. Studies have indicated that some infertility problems could be overcome through assisted reproductive technology (ART).

ART is a technology consisting of either in vitro fertilisation (IVF), intracytoplasmic sperm injection (ICSI) or artificial insemination (AI). All of these techniques require viable and quality sperm to be available when contraception is required. To ensure that the cells are available, a very low temperature storage technique known as cryopreservation has been adopted. Although this technique allows sperm to be obtained and stored immediately and indefinitely when required, studies have shown that it decreases sperm quality with every thaw. Considering most males of infertile couples initially have low sperm quality and counts, utilizing cryopreservation would cause even lower quality after their sperm have already been frozen and thawed.

ICSI requires one sperm to produce fertilization. Studies have shown that sperm from infertile males who have 
undergone cryopreservation have a high prevalence of DNA and physical damage. Statistical data have supported this observation, which has shown that $85-90 \%$ of repeated pregnancy losses were attributable to genetic problems. ${ }^{2}$ Interestingly, DNA damage in sperm has been found to be related to the increasing age of the male partner. ${ }^{3}$ This in turn would make ART a non-viable option for these groups of infertile couples. Again, for couples opting to utilize ART, sperm cryopreservation must be improved.

Cryopreservation is a technique that involves a very wide range of temperature changes. There has been much speculation as to the pathophysiology of this technique, but most cryobiologists attribute the complications to osmotic changes and membranes damage, a component of cold shock injury. As such, the complications are due to the production of large quantities of free radicals during the freezing and thawing of the sperm. Free radicals are reactive oxygen and nitrogen species that are unstable due to the presence of unpaired electrons on its outer valency. Due to this instability, the molecule tends to abstract another electron from other nearby molecules. Once an electron has been stolen, the molecular structure will reorganize, become nonfunctional, and will eventually disassemble. Examples of the types of molecules that undergo this fate are lipids, proteins and DNA.

Alpha lipoic acids (ALA) have been reported to have additional functions by which they are able to regenerate vitamin $\mathrm{C}$ from reduced vitamin $\mathrm{C}$ in the presence of glutathione. This capability would allow more antioxidants to be present within the biological system without requiring increased vitamin $C$ in the sperm media and thus shifting semen media to an acidic condition. Addition of ALA into the media does not cause a major shift of $\mathrm{pH}$ into acidic regions because the acid is categorized as a weak acid. Recent findings have also indicated that ALA is able to enter the Krebs cycle, thus assisting in the production of ATP, which is required in viable sperm.

Based on the above facts, the present study was conducted to study the effect of ALA in DNA damage and in improving sperm quality during cryopreservation. As such, the results of this study may have wider clinical importance in fertility clinics and laboratories.

\section{MATERIALS AND METHODS}

Sexually mature (more than two years old) male Boer buck cross-species were selected from the Malaysian Agricultural Research and Development Institute (MARDI). Animals were housed in a normal barn and provided access to a normal goat diet and tap water. All investigations were conducted in accordance with the guiding principle for the care and use of research animals from MARDI. Fresh semen samples were collected from male adult Boer buck cross-species through the use of the artificial vagina method. Semen samples were diluted in tris-citric egg yolk extender at a ratio of 1:45 (semen:extender) and placed into $0.25 \mathrm{ml}$ sperm straws. The straws were then placed horizontally above the liquid nitrogen surface. After a freezing period of about seven minutes in the nitrogen vapour, the straws were plunged into nitrogen liquid and stored. For thawing, straws were immersed for five seconds in a water bath at $35^{\circ} \mathrm{C}$.

Sperm count and motility were performed using the Mtrack J. Imaging System and Weber Sterility Chamber. A total of 10 ul of diluted samples was put into the chamber. Motility and count were evaluated using a light microscope connected to the video monitor under magnification of $100 \mathrm{X}$ and 400X. At least 10 grids of the chamber were counted during each observation, and all procedures were performed in triplicate. Sperm were then graded based on four groups according to intensity of movement. The grades were fast progressive (A), slow progressive (B), non-progressive (C) and immotile (D). Sperm baseline readings were performed before and after one-hour incubations with ALA. This step provided a dose- response curve for ALA before proceeding with the incorporation of ALA into the extender. Incubation with ALA was carried out for one-hour, using an ELISA bottom-flat plate in $37^{\circ} \mathrm{C}$. A total of $100 \mathrm{ul}$ diluted sperm was put into the plate according to the different concentrations of ALA used, i.e., 0.1, 0.05, 0.025, 0.0125 and $0.00625 \mathrm{mmol} /$ $\mathrm{ml}$. Graphs for motility and viability rate versus different concentrations of ALA were plotted.

DNA damage was assessed using a modified alkaline comet assay as described by Singh et al. $(2003)^{3}$. Slide preparations for the alkaline assay consisted of low (LMA) and normal (NMA) melting agar. Both gel mixtures were kept at $37^{\circ} \mathrm{C}$ to maintain a liquid state until use. Slides were prepared by first applying a thin layer of $0.75 \%$ NMA to fully frosted slides to ensure adhesion of subsequent layers. A 100 ul drop of the agarose was spread across the length of the slides with a coverslip. Any excess was scrapped off. Then, the slides were allowed to solidify for 15 minutes at $4^{\circ} \mathrm{C}$. The top layer was made by mixing $75 \mathrm{ul}$ of LMA with $10 \mathrm{ul}$ of sperm suspension. A volume of 85 ul was then pipetted onto the first layer, covered with a coverslip and allowed to solidify for another 15 minutes at $4^{\circ} \mathrm{C}$.

All slide incubation buffers mentioned below were freshly prepared. The slides that had been created previously were gently immersed in a Coplin jar containing pre-chilled lysing buffer for one hour. The slides were then removed from the solution and placed in a horizontal electrophoresis tank. The slides were then equilibrated with freshly-made electrophoresis solution, left for 20 minutes to allow the 
DNA to unwind. Electrophoresis was conducted for 10 minutes at $25 \mathrm{~V}$ and $300 \mathrm{~mA}$. Subsequently, the slides were then drained and flooded with three 15-minute changes of fresh neutralization solution and air-dried. The dried slides were stained with ethidium bromide by placing them in the staining solution $(20 \mathrm{ug} / \mathrm{ml})$. To ensure the staining was even, the slides and the solution were put on a Belly Dancer (Rocky 3D, GmbH, Germany).

Spermatozoa analyzed for comets were visualized under a fluorescence microscope. Whole sperm heads without a comet were considered undamaged, whereas spermatozoa with fragmented DNA that had migrated from the sperm head, causing a comet pattern, were considered damaged. A total of 25 sperm cells per slide were assessed for comets. The comets were captured with a video camera connected to the fluorescent microscope, and the images were evaluated quantitatively for the percentage of tail DNA using a comet assay imaging software (Comet Assay Software Project, CASP version 2, Free Software Foundation. Inc.)

\section{Statistical Analysis}

All analyses were performed using SPSS software version 12. All values obtained were tested against a normality test before analysis for the comparison between means. Since the number (N) of data samples was less than 100, Shapiro-Wilks was chosen as the normality test. Data were not normally distributed $(\mathrm{p}<0.05)$, so Kruskall Wallis ANOVA was chosen to compare the effect of different concentrations of ALA versus the percentage of DNA damage. The Mann-Whitney test was used to compare the effect of different concentrations of ALA after the incubation period on sperm motility and vitality. All values obtained were presented as the mean \pm SEM or median where appropriate. A probability of $\mathrm{p}<0.05$ was deemed a significant difference in each case.

\section{RESULTS}

The mean values of fresh or baseline buck semen were within the range of normozoospermia based on WHO (1999) recommendations, as shown in Table 1.

There was a significant change in sperm motility in all concentration used. However, the percentages of changes were different for each of the concentration groups. The percentage of motile sperm increased dramatically, from $112.8 \%$ (concentration of $0.00625 \mathrm{mmol} / \mathrm{ml})$ to $251.0 \%(0.0125 \mathrm{mmol} /$ $\mathrm{ml})$. The increasing phase pattern change reversed following introduction of more concentrated solutions of ALA. This is clearly shown in the graph in Figure 1.

The changes in sperm motility based on the different
Table 1 - Sperm motility grading in this study compared to WHO (1999) recommendation

\begin{tabular}{|c|c|c|}
\hline $\begin{array}{l}\text { Parameter } \\
\text { Density }\end{array}$ & Values & WHO (1999) criterion \\
\hline 1.Grade A & $26.40 \pm 1.75 \%$ & \multirow{4}{*}{$\begin{array}{l}50 \% \text { of forward movements (Grade } \\
\text { A \& B) or } 25 \% \text { or more Grade A for- } \\
\text { ward movement within } 60 \text { minutes } \\
\text { of ejaculations }\end{array}$} \\
\hline 2. Grade B & $41.99 \pm 1.98 \%$ & \\
\hline 3. Grade C & $18.25 \pm 0.65 \%$ & \\
\hline 4. Grade D & $13.35 \pm 0.15 \%$ & \\
\hline Motility & $\begin{array}{l}2.99 \pm 0.49 \mathrm{um} / \mathrm{sec} \\
\text { or } 68.40 \pm 0.53 \%\end{array}$ & $\begin{array}{l}\text { More than } 8 \mathrm{um} / \mathrm{sec} \text { or more than } \\
50 \% \text { motile }\end{array}$ \\
\hline Viability & $86.53 \pm 0.11 \%$ & More than $75 \%$ viable \\
\hline
\end{tabular}

The grading was done using Weber Sterility chamber under light microscope. Grade A - fast progressive movement; Grade B - slow progressive movement; Grade C - non - progressive movement; Grade D - immotile; $*$ motility percentage $=$ Grade $\mathrm{A}+$ Grade $\mathrm{B} ; * *$ viability percentage $=$ Grade $\mathrm{A}+$ Grade B + Grade C.

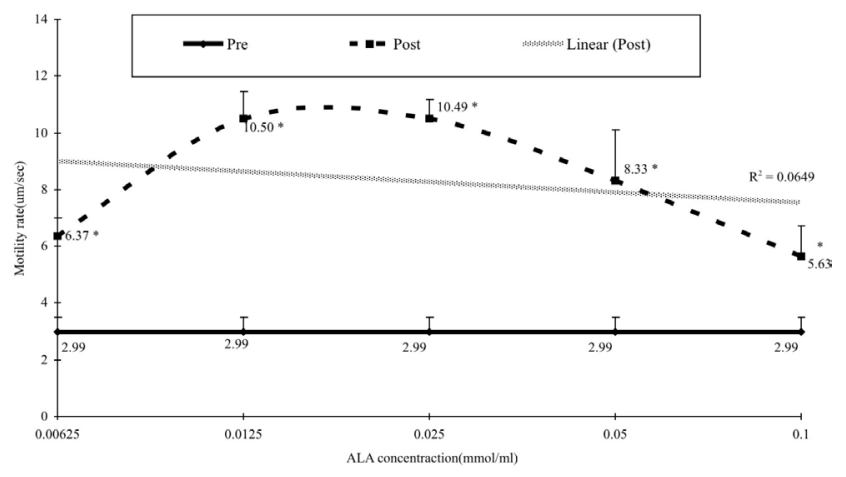

Figure 1 - Dose response curve of ALA against sperm motility. Sperm motility was assessed using light microscope with Mtrack J Imaging System on the Weber sterility chamber. Mann-Whitney t-test was used to determine the differences between baseline (pre) and after one-hour incubation (post). $\mathrm{r}^{2}=0.0649 * \mathrm{P}<0.05 . \mathrm{N}=31$

concentrations of ALA after the incubation period of one hour are shown in Figure 1. The results show that sperm motility increases following an increase in ALA concentration, until $0.025 \mathrm{mmol} / \mathrm{ml}$ is reached. Then, sperm motility decreased, even though the ALA concentration increased. These findings suggest that an optimal concentration of ALA is important in determining sperm motility.

It was also observed that sperm viability changes after a one-hour incubation with ALA (in Figure 2). While there was no significant change, the graph clearly showed that the viability rate was inversely proportional to the increment in ALA concentration.

In Figure 3, the DNA tail moment using the comet assay in sperm cells after a one-hour incubation with ALA is shown. The assay involved a negative control and tests. Based on the Kruskall Wallis ANOVA, there were significant changes between ALA concentration and average score in 


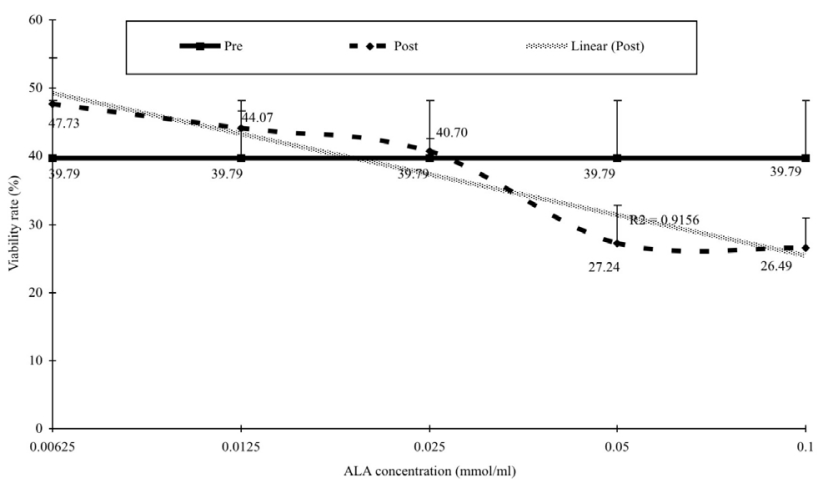

Figure 2 - Dose response curve of ALA against sperm vitality. Sperm vitality was assessed using light microscope with Mtrack J Imaging System on the Weber sterility chamber. Mann-Whitney t-test was used to determine the differences between baseline (pre) and after one-hour incubation (post). There is no significant differences between pre and post treatment $(\mathrm{P}>0.05)$. $\mathrm{r}^{2}=0.9156 \mathrm{~N}=31$

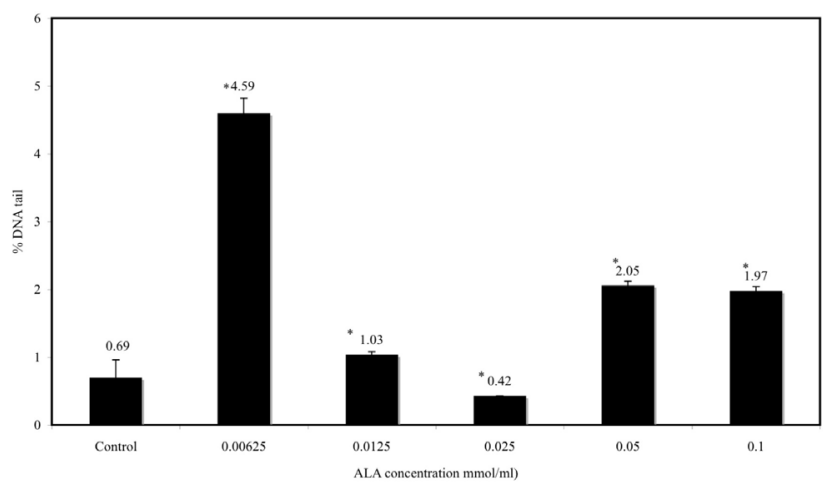

Figure 3 - Percentage of tail DNA ( $N=31$ ) against a range of ALA concentration $(0,0.00625,0.0125,0.025,0.05$ and $0.1 \mathrm{mmol} / \mathrm{ml})$. Percentage of tail DNA was done using Comet assay imaging software (CASP version 2). Kruskal Wallis ANOVA was used to determine significant differences between ALA concentrations and percentage of DNA tail. There is a significant differences between the range of concentration compared to control group. $* \mathrm{P}<0.05$

DNA tail moment. This study thus showed that incubation with ALA affected the DNA tail moment. The optimal concentration for ALA was identified as $0.025 \mathrm{mmol} / \mathrm{ml}$.

\section{DISCUSSION}

DNA peroxidation is a process that involves a chemical reaction of free radicals with the nucleotide component of sperm DNA. This reaction has a cascading effect and was found to be responsible for nucleotide deletion, insertion, frame shift, DNA nicks and DNA fragmentation. In order to minimize free radical activities, use of an antioxidant to suppress free radical formation during freezing is essential. Antioxidants are defined as substances that protect molecules from oxidation by being oxidized themselves. Currently, there are many types of antioxidants. Vitamin E, C and A are classified as non-enzymatic antioxidants, while superoxide dismutase, catalase, glutathione and alpha lipoic acids are grouped as enzymatic antioxidants. Enzymatic antioxidants are generally efficient free radical scavengers, but their activities are largely isolated to free radicals present in the aqueous phase. An exception to this limitation is ALA, which is said to be a universal antioxidant because it is composed of water and lipid-soluble antioxidant.

Results of the experiments showed that the percentage of motile sperm increased dramatically, from $112.8 \%$ (0.00625 $\mathrm{mmol} / \mathrm{ml})$ to $251.0 \%(0.0125 \mathrm{mmol} / \mathrm{ml})$. This implies that increasing ALA one-fold would also improve sperm motility with similar effect. Unfortunately, the increasing pattern changed following the introduction of more concentrated solutions of ALA (Figure 1). This would suggest that, even though ALA is capable of significantly improving the sperm motility rate, adding more ALA into the media would cause the media $\mathrm{pH}$ to become slightly acidic, thus killing the sperm.

Sperm mobility is largely dependent upon three major factors - regulation, structural integrity and energy supply. Regulation of movements is controlled at the mid-piece, particularly the flagellar and principal area. Each of these locations handles a unique function of sperm movement. The flagellar mid-piece controls the activation of motility, while the principal mid-piece handles hyperactivation. ${ }^{4}$ The unsaturated lipid content and saturated protein channels in the mid-piece usually make it the first choice for free radical attack. Addition of ALA into the extender media allows the antioxidant (ALA) to protect these components by creating a shield surrounding the mid-piece (aqueous layer) and within the structure itself (lipid layer). ${ }^{5}$

The ability of ALA to create a robust shield on the cell membrane, along with the liquid that surrounds the sperm indirectly, enhance the ability of the sperm to tolerate higher volumes of free radical attack. This ability will, in turn, indirectly reduce formation of deep pores and cracks on the sperm surface, thus ensuring structural integrity. The rate of sperm movement is largely dependent on the availability of its energy supply. Due to this, normal active sperm usually have a very active functioning mitochondria, which in turn generates high quantities of free radicals as a by-product. To ensure constant generation of ATP, external and internal structural integrity of the organelle must be maintained. Since the membrane wall and the various compartments of the organelle are high in lipid content, addition of ALA would protect these structures from the ever-increasing free radical species, which are a by-product of the Krebs cycle.

Sperm mitochondria ability is also dependent on the availability of ATP-based enzymes. Addition of ALA is thought to have assisted in the metabolism of oxidative 
decarboxylation by acting as a co-enzyme. ${ }^{6}$ The increase in oxidative decarboxylation would increase cytochrome $\mathrm{C}$ concentration and thus directly increase the mitochondria's membrane potential, improving regulation of mitochondria function and its biogenesis. ${ }^{7}$ In addition to the above, ALA has also been reported to assist the mitochondria's citric cycle. This in turn will increase the level of reduced glutathione, ATP, TCA cycle enzyme and electron transport chain complex activities. ${ }^{8}$ ALA regulation of metabolism, increased availability of mitochondrial co-enzymes and improvement of protection of free radicals are thought to eventually lead to a reduced incidence of mitochondria dysfunction, thus ensuring sufficient ATP for sperm movement. ${ }^{9}$

Currently, sperm quality is determined through its morphology and motility. Although this method had been adopted by various fertility laboratories and is considered a gold standard, recent reports have suggested that other factors must also be taken into consideration..$^{10}$ Recent findings have suggested that the DNA fragmentation index (DFI) was higher in idiopathic infertile men with the normal routine semen parameters than in fertile male subjects. ${ }^{11}$ If conception is ever achieved by these idiopathic infertile men, it is associated with repeated early fetal loss, and the prevalence of incomplete pregnancies is greater than normal. ${ }^{12}$ To ensure that full conception is achieved, assessment of sperm DNA fragmentation is required. Until now, the best method for this assessment was the use of the comet assay, since it has an ability to qualitatively identify single and double-stranded DNA damage. This study was able to confirm the fact that addition of ALA was able to minimize DNA damage.

Our study has shown that the DNA damage score was significantly lower than in the negative control. This is in accordance with a past study that had found that administration of ALA to rats prevented oxidative stress and decreased DNA strand breaks. ${ }^{13}$ Based on the results, it can be concluded that there was significant correlation between sperm motility and DNA damage. Addition of ALA into the extender media has been shown to suppress DNA damage, particularly at $0.025 \mathrm{mmol} / \mathrm{ml}$.

Viable sperm by definition include even those that are non-progressively motile. Sperm viability is closely associated with regulated homeostasis and sperm membrane integrity. As mentioned above, unregulated free radical activity resulting from either environmental stress or inadequate supply of antioxidants would disrupt membrane structure. The disruption of the lipid and protein components would eventually lead to selective permeability capabilities loss and, eventually, cell death. Even though ALA is known to posses potent anti-oxidative properties, the addition of ALA into the sperm environment would shift the $\mathrm{pH}$ towards acidity. Studies have shown that the speed of sperm immobilization is proportional to the activity of hydrogen ions in the sperm's environment. Sperm will be non-motile and enter apoptosis if the $\mathrm{pH}$ of its environment is not maintained within $\mathrm{pH} 4.0$ and 7.5. ${ }^{14}$ These facts are clearly depicted in this study, in which high concentrations of ALA caused a dramatic reduction in potentially viable sperm.

Although ALA is recognized as a universal antioxidant, with abilities to scavenge free radicals in aqueous and nonaqueous phases, its main efficacy is derived from its ability to minimize peroxynitrite-induced damage efficiently. This indirect efficiency is attributed to its ability to regulate glutathione-s-transferase (GST) activity. ${ }^{15}$ Through the upregulation of this enzyme, cellular and organelle membranes would be protected from synthetic peroxides, oxidized lipids, retinoids, and the cytotoxicity of both 4-hydroxy-2-nonenal (4-HNE) and hydrogen peroxide. ${ }^{16,17}$

Earlier studies have conclusively indicated that hydrogen peroxide is the main reactive oxygen species involved in induced motility loss, impairment of sperm-oocyte fusion and acrosomal exocytosis. ${ }^{18}$ Hence, a significant reduction of this component would in turn enhance sperm viability. Interestingly, a past study also found that lipoic acid may act as a cryoprotectant while being administered one day prior to adriamycin therapy, thus maintaining normal steroidogenesis and spermatogenesis. ${ }^{19}$

Recently, different techniques, including COMET assay, Terminal transferase dUTP Nick End Labelling (TUNEL) and sperm chromatin structure assay (SCSA), have been noted to detect DNA damage. ${ }^{20}$ The present study can open the door for future studies to detect DNA damage in sperm using ALA.

Results obtained from this study show that an increase in sperm motility was caused by ALA capabilities in energy production but that decreases could be caused by the acidity of the environment that produces immobilization. This was further supported by previous findings, which suggested that sperm motility is severely compromised when acidity is induced by $\mathrm{HCl}$.

\section{CONCLUSION}

It should be noted that damage to sperm DNA may adversely affect male fertility and contribute to poorer embryo development and lower pregnancy rates. ${ }^{21}$ Proper knowledge of the causes of DNA damage and the various factors related to its integrity may be helpful in treating infertility. In summary, based on the results of our study, it can be concluded that an optimal concentration of ALA was able to improve sperm motility and viability and minimize DNA damage. 


\section{REFERENCES}

1. Isidori A, Latini M, Romanelli F. Treatment of male infertility. Contraception. 2005; 72:314-8.

2. Tesarik J, Greco E, Mendoza C. Late, but not early, paternal effect on human embryo development is related to sperm DNA fragmentation. Hum Reprod. 2005;19:611-5.

3. Singh NP, Muller CH, Berger RE. Effects of age on DNA double-strand breaks and apoptosis in human sperm. Fertil Steril. 2003;80:1420-30.

4. Suarez SS, Marquez B, Harris TP, Schimenti JC. Different regulatory systems operate in the midpiece and principal piece of the mammalian sperm flagellum. Soc Reprod Fertil Suppl. 2007;65:331-4.

5. Hamano Y. Continuous infusion of lipoic acid rapidly reduces plasma beta-hydroxybutyrate with elevation of non-esterified fatty acids in broiler chickens. Br J Nutr. 2007; 97:495-501.

6. Plotnikov EY, Kazachenko AV, Vyssokikh MY, Vasileva AK, Tcvirkun $\mathrm{DV}$, Isaev NK, et al. The role of mitochondria in oxidative and nitrosative stress during ischemia/reperfusion in the rat kidney. Kidney Int. 2007;72:1493-502.

7. Gopalakrishnan L, Scarpulla RC. Differential regulation of respiratory chain subunits by a CREB-dependent signal transduction pathway. Role of cyclic AMP in cytochrome c and COXIV gene expression. J Biol Chem. 1994;269:105-13.

8. Palaniappan AR, Dai A. Mitochondrial ageing and the beneficial role of alpha-lipoic Acid. Neurochem Res. 2007;32:1552-8.

9. Moreira PI, Harris PL, Zhu X, Santos MS, Oliveira CR, Smith MA et al. Lipoic Acid and N-acetyl Cysteine Decrease Mitochondrial-Related Oxidative Stress in Alzheimer Disease Patient Fibroblasts. J Alzheimers Dis. 2007;12:195-206.

10. Tarozzi N, Bizzaro D, Flamigni C, Borini A. Clinical relevance of sperm DNA damage in assisted reproduction. Reprod Biomed Online. 2007;14:746-57.

11. Sharma RK, Said T, Agarwal A. Sperm DNA damage and its clinical relevance in assessing reproductive outcome. Asian J Androl. 2004;6:139-48
12. Bhattacharya SM. Association of various sperm parameters with unexplained repeated early pregnancy loss-which is most important? Int Urol Nephrol. 2007; Sep 26 [Epub ahead of print]

13. Sundaram K, Panneerselvam KS. Oxidative stress and DNA single strand breaks in skeletal muscle of aged rats: role of carnitine and lipoicacid. Biogerontology. 2006; 7:111-8.

14. Olmsted SS, Dubin NH, Cone RA, Moench TR. The rate at which human sperm are immobilized and killed by mild acidity. Fertil Steril. 2000;73:687-93

15. Rezk BM, Haenen GR, van der Vijgh WJ, Bast A. Lipoic acid protects efficiently only against a specific form of peroxynitrite-induced damage. J Biol Chem. 2004; 279:9693-7.

16. Maeda A, Crabb JW, Palczewski K. Microsomal glutathione S-transferase 1 in the retinal pigment epithelium: protection against oxidative stress and a potential role in aging. Biochemistry. 2005;44:480-9.

17. Yang Y, Yang Y, Trent MB, He N, Lick SD, Zimniak P et al. GlutathioneS-transferase A4-4 modulates oxidative stress in endothelium: possible role in human atherosclerosis.” Atherosclerosis. 2004;173:211-21.

18. Baker MA, Aitken RJ. Reactive oxygen species in spermatozoa : methods for monitoring and significance for the origins of genetic disease and infertility. Reproductive Biology and Endocrinology. 2005;3:67-82.

19. Prahalathan C, Selvakumar E, Varalakshmi P. Lipoic acid modulates adriamycin-induced testicular toxicity. Reprod Toxicol. 2006;21:54-9.

20. Shamsi MB, Kumar R, Dada R. Evaluation of nuclear DNA damage in human spermatozoa in men opting for assisted reproduction. Indian $\mathbf{J}$ Med Res. 2008;127:115-23.

21. Meeker JD, Singh NP, Hauser R. Serum Concentrations of Estradiol and Free T4 Are Inversely Correlated with Sperm DNA Damage in Men from an Infertility Clinic. J Androl. 2008 Mar 20 [Epub ahead of print]. 